\title{
Research of a Novel Visual Tracking System Based on a Ring Type Piezoelectric Actuator
}

\author{
Xinda Qi ${ }^{1, a}$, Shengjun Shi, ${ }^{1, b}$, Yingxiang Liu ${ }^{1}$, Huaiyin Xiong ${ }^{1}$ and Weishan Chen ${ }^{1}$ \\ ${ }^{1}$ Department of Mechatronic Engineering, Harbin Institute of Technology, West Da-Zhi Street, Harbin, \\ China \\ axinda.qi@outlook.com, ${ }^{\mathrm{b}}$ sirssj@hit.edu.cn \\ *corresponding author
}

Keywords: piezoelectric actuator, tracking strategy, dead zone compensation, variable parameter PID controller, visual tracking system.

\begin{abstract}
This study proposes a target tracking system based on a multi-DOF piezoelectric actuator in order to achieve fast and accurate target tracking because it is difficult for traditional tracking system with electromagnetic motor to behave outstanding, especially in low speed condition. The proposed piezoelectric actuator uses hybrid vibration consisted of three modes to make four driving feet rotating bionic eye in three directions. Based on images collected from camera, the bionic eye can track targets object through particle filter tracking algorithm. The tracking system's angle error is less than 0.5 degree when tracking a sine movement of $1 \mathrm{~Hz}$ and 10 degrees' amplitude. The tracking error is controlled within 5 pixels after $0.22 \mathrm{~s}$ and within 34 pixels when the system is tracking a step signal and a moving target with a speed of $32 \mathrm{pixel} / \mathrm{s}$, respectively.
\end{abstract}

\section{Introduction}

In recent years, the magnificent influence of tracking system on unmanned vehicle driving, auto assembling and traffic controlling becomes remarkable, which demands more researches and studies. Visual tracking system is a research hotspot, which involves automation control and image processing [1]. It uses camera as information collector which makes it become EMI free and elusive. Besides, visual tracking system can provide more location and motion information about targets than most of other transducers, which makes target tracking robust and reliable [2-3].

Most mature techniques of visual tracking systems use a structure of multiple-axis PTZ with electromagnetic motors, whose complex structure and motor reducer decrease tracking speed and accuracy of the system. To deal with this problem, visual tracking systems based on bionic eye were developed rapidly and it could provide enough and timely information for robots. Bionic eye with axes driven by DC motors was developed at first [4]. The structure was based on three axes and each axis was driven by one motor, which was large and difficult to embed in robots. After that, bionic eyes with artificial muscles were developed [5]. The volume of the visual tracking system decreased and the structure became more compacted.

The tracking system based on bionic eyes has several advantages compared with the traditional moving camera. Firstly, the volume is smaller and it is easier to embed in various robots. Secondly, the compacted structure has less angular momentum when moved, which can help improving the dynamic characters and response speed. Thirdly, the system needs less energy to operate. However, the traditional visual systems with bionic eyes also have limitations. Firstly, the bionic eyes use parallel mechanism or axes with motors to rotate, whose gaps influence control effect. Furthermore, the structure of driving mechanism is complex and difficult to fabricate.

Piezoelectric actuator is relatively small and it contacts with mover tightly, so the eye can be rotated by it accurately and is convenient to embedded. It also responds quickly to signals because of direct driving. Then a tracking system based on piezoelectric actuator and bionic eye can be designed through this idea, which is possible to behave well in tracking. Altogether the advantages 
of novel visual tracking system can be anticipate: higher accuracy, less error and smaller volume.

\section{Mechanism of Tracking Device}

In order to achieve fast and accurate target tracking, a driving mechanical system is needed and a multiple degrees of freedom piezoelectric actuator is chosen to drive the device. This research uses a ring type multi-DOF piezoelectric actuator to drive a bionic eye directly, as shown in Figure 1. The bionic eye uses steel shell, which can press the actuator tightly via a magnetic field produced by the permanent magnet embedded in the substrate. Twelve piezoelectric pieces are located on the upper side of the actuator's base structure and a whole round piece of piezoelectric ceramic adheres to the back side of the base. Each ceramic has an electrode on its outer surface and is grounded via the metal base. Four driving feet on the base are used to rotate the eye when vibrating.

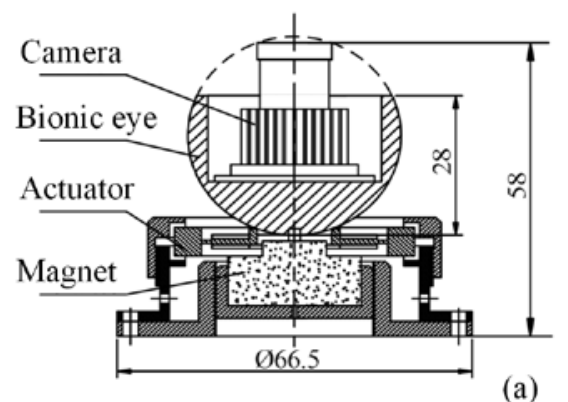

(a)

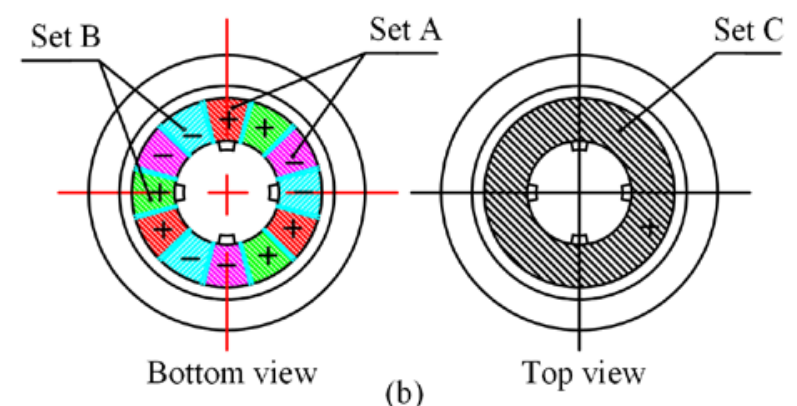

(b)

Figure 1 The tracking device: (a) the structure of tracking device; (b) actuator's structure.

The operation of the multi-DOF actuator is based on three bending vibration modes. The four driving feet vibrate in different directions in different modes, as shown is Figure 2(a), and combinations of different modes let the feet have elliptical tracks in different planes. When bionic eye contacts the actuator, the four feet can rotate the bionic eye around three axes due to the elliptical vibrations. Meanwhile, the rotation speed and direction are influenced by the vibration modes and their extents, which are decided by AC excitation signals. The resonant frequency of the modes has been degenerated and tested to be $37 \mathrm{kHz}$, the same as the excitation signals' frequency.

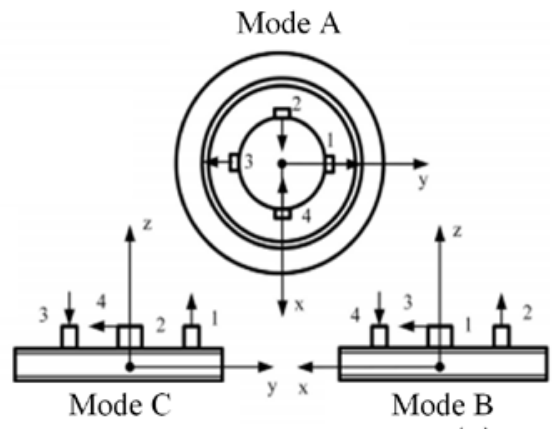

(a)

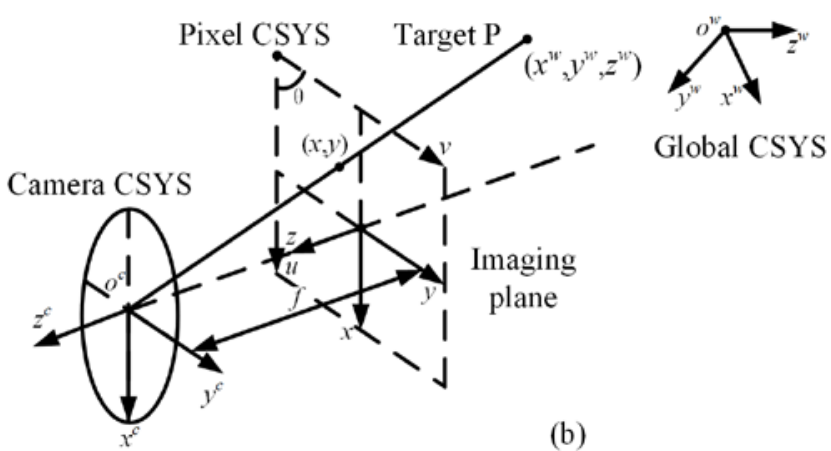

(b)

Figure 2 Working mechanisms: (a) vibration modes of the actuator; (b) model of camera imaging.

\section{Visual Tracking Strategy}

Came from the tracking device of the system, images are processed to produce the target's location data, which is a promise of tracking strategy. Thus, the camera needs to be calibrated at first to obtain parameters and transform matrix for restoration of the target's location .

Based on convex lens imaging method, a model of camera imaging is developed, as shown in Figure 2(b). For convenience, the upside down real image is placed in front of the lens.

After a analysis and simplification of the transformation between the camera CSYS and the pixel CSYS, a transformation equation is calculated and shown in equation (1). The equation includes scale, translate and rotate transformation, which are produced by convex imaging, origin 
malposition and arrangement error. Based on this equation, space locations of the target can be restored from the collected images after the equation parameters are calculated through the images of standard pattern which are shot in various angles.

$$
\left[\begin{array}{c}
u \\
v \\
1
\end{array}\right]=R_{c}^{p}\left[\begin{array}{c}
X^{c} \\
Y^{c} \\
1
\end{array}\right]=\left[\begin{array}{ccc}
1 / d_{x} & -\cot \theta / d_{x} & u_{0} \\
0 & 1 /\left(d_{y} \sin \theta\right) & v_{0} \\
0 & 0 & 1
\end{array}\right]\left[\begin{array}{c}
X^{c} \\
Y^{c} \\
1
\end{array}\right]
$$

In which $u$ and $v$ are coordinates of the target in X and Y direction of pixel CSYS. $d_{x}$ and $d_{y}$ are pixel's lengths in $\mathrm{X}$ and $\mathrm{Y}$ direction. $X^{c}$ and $Y^{C}$ are the coordinates of the target in $\mathrm{X}$ and $\mathrm{Y}$ direction of camera CSYS. $\theta$ is the angle between two axes of pixel CSYS. $u_{0}$ and $v_{0}$ are the distances of origin malposition in $\mathrm{X}$ and $\mathrm{Y}$ direction, respectively.

Besides, the distortion condition should be calibrated before target tracking to rectify distortion errors and a model of camera distortion is introduced:

$$
\left\{\begin{array}{l}
X_{d}^{c}=X^{c}\left(1+k_{c 1} r^{2}+k_{c 2} r^{4}+k_{c 5} r^{6}\right)+2 k_{c 3} X^{c} Y^{c}+k_{c 4}\left(r^{2}+2 X^{c^{2}}\right) \\
Y_{d}^{c}=Y^{c}\left(1+k_{c 1} r^{2}+k_{c 2} r^{4}+k_{c 5} r^{6}\right)+2 k_{c 4} X^{c} Y^{c}+k_{c 3}\left(r^{2}+2 Y^{c^{2}}\right)
\end{array}\right.
$$

In which $r^{2}=X^{c 2}+Y^{c 2} .\left(X_{d}^{c}, Y_{d}^{c}\right)$ are the target coordinates after camera distortion. $k_{c 1}, k_{c 2}, k_{c 3}$, $k_{c 4}$ and $k_{c 5}$ are distortion factors.

The calibration of the camera is based on the images of a checker broad pattern in different shooting angles, as shown in Figure 3(a). Using MATLAB, parameters of equations are calculated and they are used to extract the image's space information.

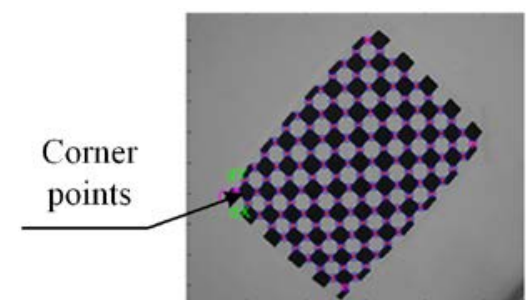

(a)

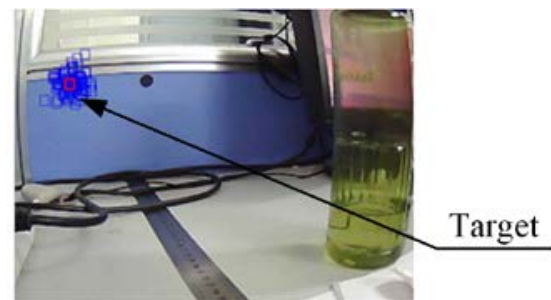

(b)

Figure 3 Visual tracking experiments: (a) calibration of camera; (b) visual tracking result.

With the target's location data came from the camera, a particle filter algorithm is used to recognize and track the target patterns from the background of pictures, which uses abundant weight based particles to simulate probability distribution functions. Firstly, a set of the particles is created and expressed as:

$$
\left\{\begin{array}{l}
S=\left\{\left(\mathrm{s}^{(\mathrm{n})}, w^{(\mathrm{n})}\right) \mid \mathrm{n}=1 \ldots N\right\} \\
\sum_{n=1}^{N} w^{(\mathrm{n})}=1
\end{array}\right.
$$

In which $s^{(\mathrm{n})}$ is the location of the particles. $w^{(\mathrm{n})}$ is the weight of particles, which is the possibility distribution of the target location. $N$ is the number of the particles.

Then, each particle' colour histogram is measured from HSV images to identify the different particles and to represent their character, because HSV method can decrease the influence of light changing. The Bhattacharyya distance $d$ is used to measure the similarity between each particle and the target and a kind of weight of particle $w^{(\mathrm{n})}$ is calculated for better expression of possibility, as shown in equation (4): 


$$
\left\{\begin{array}{l}
d=\sqrt{1-\sum_{i=1}^{m} \sqrt{p^{(\mathrm{i})} q^{(\mathrm{i})}}} \\
w^{(\mathrm{n})}=\frac{1}{\sqrt{2 \pi} \sigma} e^{-\frac{d^{2}}{2 \sigma^{2}}}
\end{array}\right.
$$

In which $p^{(\mathrm{i})}$ and $q^{(\mathrm{i})}$ are the histograms of the target and particles, respectively.

Based on the particle's weight and the location data collected from the camera, the anticipate tracking output $E(S)$ is calculated, as shown in equation (5). Thus, the tracking task of one image is complete. Furthermore, resampling of particle set and updating of colour histogram are used to improve tracking quality before returning to the first step of the tracking loop when the system is tracking moving targets. A visual tracking experiment shows that the algorithm works well and can provide reliable data for control of the tracking device, as shown in Figure 3(b).

$$
E(\mathrm{~S})=\sum_{n=1}^{N} w^{(\mathrm{n})} S^{(n)}
$$

\section{Experimental Environment Design}

From the design of the tracking device, a bionic eye and a piezoelectric actuator were fabricated and assembled, as shown in Figure 4(a). A performance test platform was designed to evaluate dynamic characters and accuracy of the tracking device, as shown in Figure 4(b). A photoeletric encoder was connected to the bionic eye to measure angular movement of it. Weights and springs were installed to change the actuator's working condition.

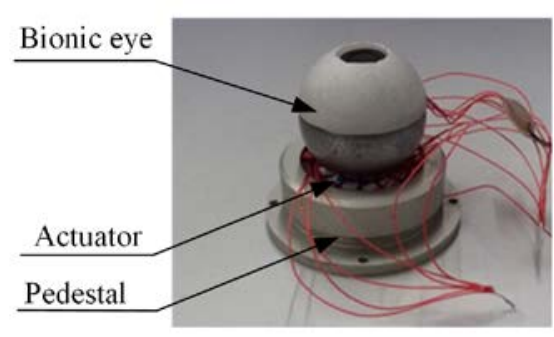

(a)

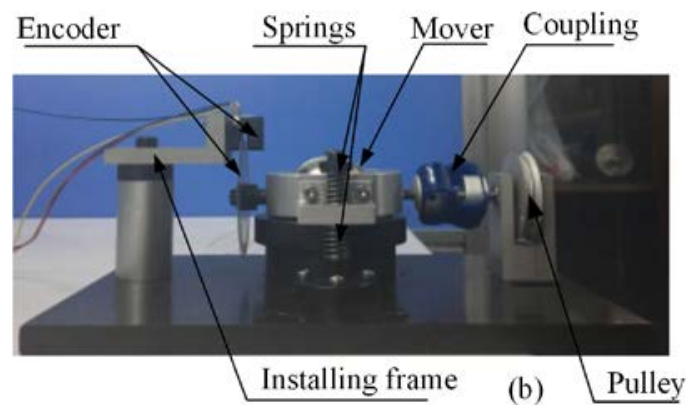

(b) Pulley

Figure 4 The experimental set-ups: (a) tracking device; (b) performance test platform.

A tracking test platform was built to test the system's tracking performance of moving targets, as shown in Figure 5. An actuator controller was designed and produced based on DSP/FPGA, which was programmed to produce controllable AC signals and achieved tracking algorithm and various control methods. All of the data and images were collected to a computer which evaluated the system's performance and tracking error.

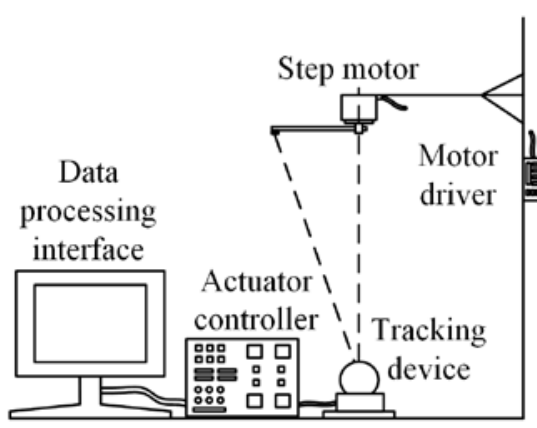

(a)

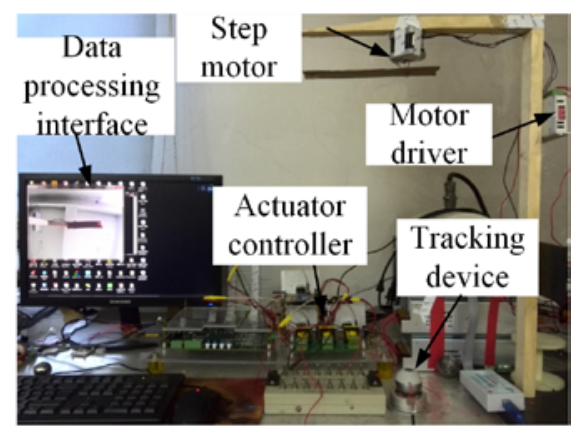

(b)

Figure 5 Tracking system and experimental set-up for moving target: (a) structure; (b) photograph. 


\section{Tracking Experiment Results and Analysis}

After setting up the experimental environment, experiments to choose proper control method were conducted. In the experiments, the PID (method 1), dead zone compensation PID (method 2) and variable structure PID with compensation (method 3) were used to control the actuator to track a $1 \mathrm{~Hz}$ sinusoidal speed signal, whose amplitude was $80 \mathrm{r} / \mathrm{min}$. Speed tracking errors were measured and calculated, as shown in Figure 6. According to the result, control method 3 was selected, because it decreased the tracking error of control method 2 by about $50 \%$ and decreased the error of method 1 by about 75\%. Control method 3's formula is expressed as:

$$
\begin{aligned}
& u(k)=K_{p}\left[e(k)+K_{i} \sum_{j=0}^{k} e(j)+K_{d}(e(k)-e(k-1))\right]+V_{\text {comp }} \operatorname{sign}\left(V_{\text {object }}\right)+k \\
& =\left[a_{p}+b_{p}\left(1-\exp \left(-c_{p}|e|\right)\right] \times\left\{e(k)+\left[a_{i} \exp \left(-c_{i}|e|\right)\right] \sum_{j=0}^{k} e(j)+\left[a_{d}-b_{d}\left(1-\exp \left(-c_{d}|e|\right)\right](e(k)-e(k-1))\right\}\right.\right. \\
& +V_{\text {comp }} \operatorname{sign}\left(V_{\text {object }}\right)+k
\end{aligned}
$$

In which $a, b, c, k$ are tuning parameters and $V_{\text {comp }}, V_{\text {object }}$ are compensation factors.
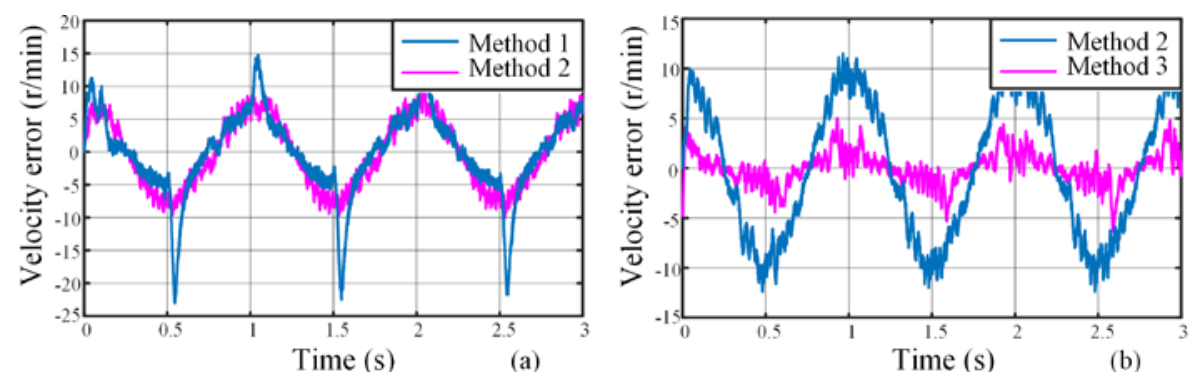

Figure 6 Velocity errors in various control methods: (a) in method 1 and 2; (b) in method 2 and 3.

There were two approaching methods based on the piezoelectric actuator to drive the bionic eye (mover): the method 1 was to rotate the mover around three axes simultaneously, the method 2 was to rotate around axes alternatively. Motion interference between axes of the method 2 was less than that of method 1 by 50\% and method 2 was selected, as shown in Figure 7.
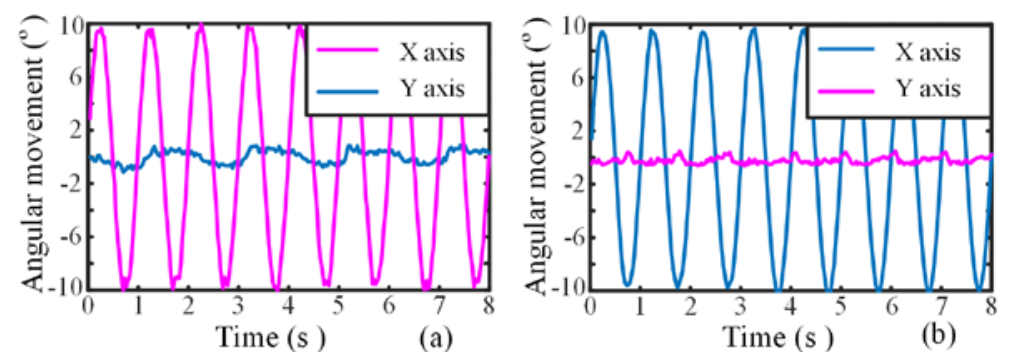

Figure 7 Interferences between two axes: (a) in approaching method 1; (b) in approaching method 2.

Based on the preparations above, several core experiments were conducted, which used the bionic eye to track a moving target. The tracking task was using tracking device to keep the target's pattern in the centre of the camera's field of view and tracking error was the distance between them. The tracking error was less than 5 pixels after 0.22s when the system was tracking a step signal of 120 pixel's amplitude, as shown in Figure 8(a), which meant the system was quick and accurate.
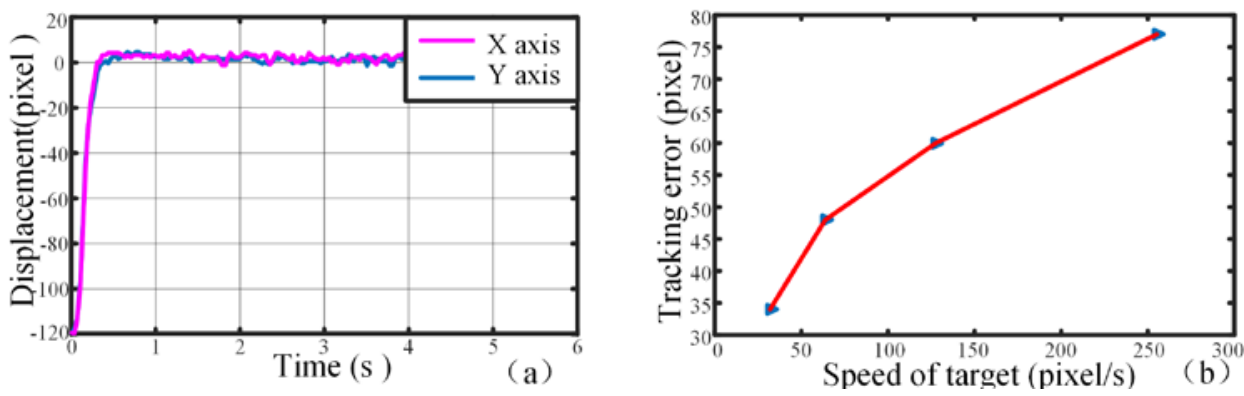
Figure 8 Tracking results: (a) tracking of a step signal; (b) tracking errors of moving target.

Based on the tracking test platform, tracking experiments on a moving target were conducted, of which the target was at the speed of $32 \mathrm{pixel} / \mathrm{s}, 64 \mathrm{pixel} / \mathrm{s}, 128 \mathrm{pixel} / \mathrm{s}$ and $256 \mathrm{pixel} / \mathrm{s}$. The error of the tracking system increased together with the target's speed, as shown in Figure 8(b). When the target's speed was less than 256 pixel/s, the camera could always catch the target and keep it within the centre area of the field of view $(320 * 320)$, as shown in Figure 9.
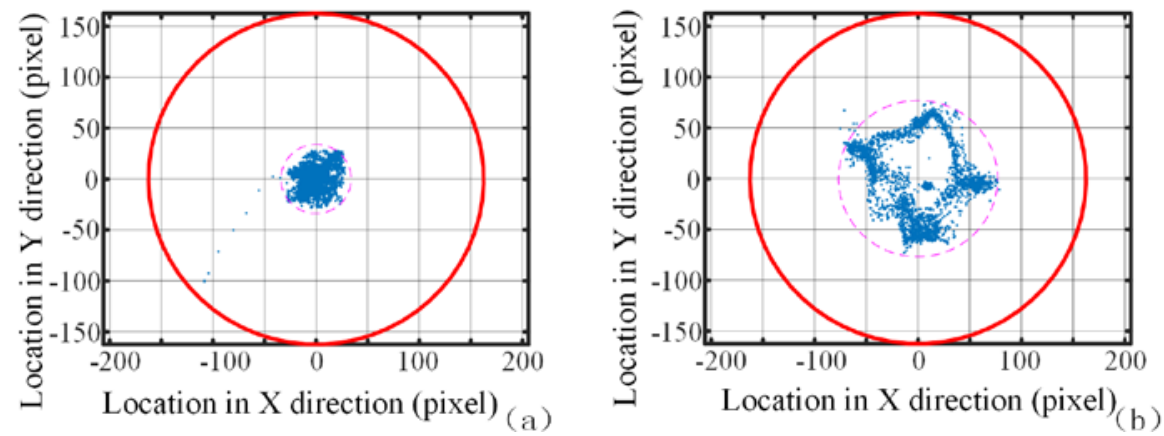

Figure 9 Target’s distributions in camera CSYS under various speeds : (a) 32 pixel/s; (b) 256 pixel/s.

These results showed that the novel visual tracking system could track the target quickly with small error after the target's pattern was recognized. Besides, the error of tracking moving target was acceptable and the target was always within the centre area of the camera's field of view when the target's speed was less than $256 \mathrm{pixel} / \mathrm{s}$, which meant that the target would not be lost and the system could work reliably in such condition.

\section{Conclusion}

This paper described the setup of a novel visual tracking system based on a piezoelectric actuator and the experiments about the system's performance. The mechanism of the tracking system was consisted of a bionic eye and a ring type piezoelectric actuator, which rotated the bionic eye through four driving feet. A tracking algorithm based on particle filter was introduced as well as a camera's imaging model, which were proved to be effective.

Several experiments were conducted to evaluate the performance of the visual tracking system. Based on related tests, the variable structure PID controller with dead zone compensation was selected because it could reduce the PID controller's error by $75 \%$. The selected approaching method was rotating around different axes alternatively because its interference between axes was about 50\% less than driving simultaneously. The tracking time for a 120 pixels' step signal was 0.22s within error of 5 pixels. When the system tracked moving targets, the errors were within 34 pixels and 77 pixels when the target's speeds were $32 \mathrm{pixel} / \mathrm{s}$ and $256 \mathrm{pixel} / \mathrm{s}$, respectively, and the tracking error increased together with the target's speed. These results show that the piezoelectric actuator can drive the bionic eye effectively and the whole visual tracking system can track targets quickly, accurately and consistently, which can be developed and utilized further in the next stage's study.

\section{References}

[1] Dickmanns, E.D. (2003) The development of machine vision for road vehicles in the last decade. Intelligent Vehicle Symposium. IEEE, vol.1, 268-281.

[2] Collins, R.T., Lipton, A.J., Kanade, T. (2000) Introduction to the special section on video surveillance. IEEE Transactions on Pattern Analysis \& Machine Intelligence, 22(22), 745-746.

[3] Wei, G.Y., Fu, L.C., Liu, D. (2001) Design and implementation of visual servoing system for realistic air target tracking. IEEE International Conference on Robotics and Automation. Proceedings. IEEE, 1(1), 229-234. 
[4] Takanishi, A., Ishimoto, S., Matsuno, T. (1995) Development of an anthropomorphic head-eye system for robot and human communication. IEEE International Workshop on Robot and Human Communication, Ro-Man'95 Tokyo, Proceedings, 77-82.

[5] Cannata, G., Maggiali, M. (2007) Design of a Humanoid Robot Eye. Humanoid Robots: New Developments, In Tech, 151-156. 